\title{
The Average Distance in a Random Graph with Given Expected Degrees
}

\author{
Fan Chung and Linyuan Lu
}

Abstract. Random graph theory is used to examine the "small-world phenomenon" any two strangers are connected through a short chain of mutual acquaintances. We will show that for certain families of random graphs with given expected degrees, the average distance is almost surely of order $\log n / \log \tilde{d}$ where $\tilde{d}$ is the weighted average of the sum of squares of the expected degrees. Of particular interest are power law random graphs in which the number of vertices of degree $k$ is proportional to $1 / k^{\beta}$ for some fixed exponent $\beta$. For the case of $\beta>3$, we prove that the average distance of the power law graphs is almost surely of $\operatorname{order} \log n / \log \tilde{d}$. However, many Internet, social, and citation networks are power law graphs with exponents in the range $2<\beta<3$ for which the power law random graphs have average distance almost surely of order $\log \log n$, but have diameter of order $\log n$ (provided having some mild constraints for the average distance and maximum degree). In particular, these graphs contain a dense subgraph, that we call the core, having $n^{c / \log \log n}$ vertices. Almost all vertices are within distance $\log \log n$ of the core although there are vertices at distance $\log n$ from the core.

\section{Introduction}

In 1967, the psychologist Stanley Milgram [Milgram 67] conducted a series of experiments which indicated that any two strangers are connected by a chain of intermediate acquaintances of length at most six. In 1999, Barabási et al. [Albert et al. 99] observed that in certain portions of the Internet, any two

(C) A K Peters, Ltd.

I542-795I/03 $\$ 0.50$ per page 
web pages are at most 19 clicks away from one another. In this paper, we will examine average distances in random graph models of large complex graphs. In turn, the study of realistic large graphs provides new directions and insights for random graph theory.

Most of the research papers in random graph theory concern the Erdős-Rényi model $\mathcal{G}_{p}$, in which each edge is independently chosen with the probability $p$ for some given $p>0$ (see [Erdős and Rényi 59]). In such random graphs, the degrees (the number of neighbors) of vertices all have the same expected value. However, many large random-like graphs that arise in various applications have diverse degree distributions [Aiello et al. 01b, Barabási and Albert 99, Albert et al. 99, Jeong et al. 00, Kleinberg et al. 99, Lu 01]. It is therefore natural to consider classes of random graphs with general degree sequences.

We consider a general model $G(\mathbf{w})$ for random graphs with given expected degree sequence $\mathbf{w}=\left(w_{1}, w_{2}, \ldots, w_{n}\right)$. The edge between $v_{i}$ and $v_{j}$ is chosen independently with probability $p_{i j}$ where $p_{i j}$ is proportional to the product $w_{i} w_{j}$. The classical random graph $G(n, p)$ can be viewed as a special case of $G(\mathbf{w})$ by taking $\mathbf{w}$ to be $(p n, p n, \ldots, p n)$. Our random graph model $G(\mathbf{w})$ is different from the random graph models with an exact degree sequence as considered by Molloy and Reed [Molloy and Reed 95, Molloy and Reed 98], and Newman, Strogatz, and Watts [Newman et al. 00]. Deriving rigorous proofs for random graphs with exact degree sequences is rather complicated and usually requires additional "smoothing" conditions because of the dependency among the edges (see [Molloy and Reed 95]).

Although $G(\mathbf{w})$ is well defined for arbitrary degree distributions, it is of particular interest to study power law graphs. Many realistic networks such as the Internet, social, and citation networks have degrees obeying a power law. Namely, the fraction of vertices with degree $k$ is proportional to $1 / k^{\beta}$ for some constant $\beta>1$. For example, the Internet graphs have powers ranging from 2.1 to 2.45 (see [Albert et al. 99, Faloutsos et al. 99, Broder et al. 00, Kleinberg et al. 99]). The collaboration graph of Mathematical Reviews has $\beta=2.97$ (see [Grossman et al. 03]). The power law distribution has a long history that can be traced back to Zipf [Zipf 49], Lotka [Lotka 26] and Pareto [Pareto 1897]. Recently, the impetus for modeling and analyzing large complex networks has led to renewed interest in power law graphs.

In this paper, we will show that for certain families of random graphs with given expected degrees, the average distance is almost surely $(1+o(1)) \log n / \log \tilde{d}$. Here $\tilde{d}$ denotes the second-order average degree defined by $\tilde{d}=\sum w_{i}^{2} / \sum w_{i}$, where $w_{i}$ denotes the expected degree of the $i$-th vertex. Consequently, the average distance for a power law random graph on $n$ vertices with exponent $\beta>3$ is almost surely $(1+o(1)) \log n / \log \tilde{d}$. When the exponent $\beta$ satisfies $2<\beta<3$, 
the power law graphs have a very different behavior. For example, for $\beta>3$, $\tilde{d}$ is a function of $\beta$ and is independent of $n$, but for $2<\beta<3, \tilde{d}$ can be as large as a fixed power of $n$. We will prove that for a power law graph with exponent $2<\beta<3$, the average distance is almost surely $O(\log \log n$ ) (and not $\log n / \log \tilde{d})$ if the average degree is strictly greater than 1 and the maximum degree is sufficiently large. Also, there is a dense subgraph, that we call the "core," of diameter $O(\log \log n)$ in such a power law random graph such that almost all vertices are at distance at most $O(\log \log n)$ from the core, although there are vertices at distance at least $c \log n$ from the core. At the phase transition point of $\beta=3$, the random power law graph almost surely has average distance of order $\log n / \log \log n$ and diameter of order $\log n$.

\section{Definitions and Statements of the Main Theorems}

In a random graph $G \in G(\mathbf{w})$ with a given expected degree sequence $\mathbf{w}=$ $\left(w_{1}, w_{2}, \ldots, w_{n}\right)$, the probability $p_{i j}$ of having an edge between $v_{i}$ and $v_{j}$ is $w_{i} w_{j} \rho$ for $\rho=\frac{1}{\sum_{i} w_{i}}$. We assume that $\max _{i} w_{i}^{2}<\sum_{i} w_{i}$ so that the probability $p_{i j}=w_{i} w_{j} \rho$ is strictly between 0 and 1 . This assumption also ensures that the degree sequence $w_{i}$ can be realized as the degree sequence of a graph if the $w_{i}$ are integers [Erdős and Gallai 59]. Our goal is to have as few conditions as possible on the $w_{i}$ while still being able to derive good estimates for the average distance.

First, we need some definitions for several quantities associated with $G$ and $G(\mathbf{w})$. In a graph $G$, the volume of a subset $S$ of vertices in $G$ is defined to be $\operatorname{vol}(S)=\sum_{v \in S} \operatorname{deg}(v)$, the sum of degrees of all vertices in $S$. For a graph $G$ in $G(\mathbf{w})$, the expected degree of $v_{i}$ is exactly $w_{i}$ and the expected volume of $G$ is $\operatorname{Vol}(G)=\sum_{i} w_{i}$. By the Chernoff inequality for large deviations [Alon and Spencer 92], we have

$$
\operatorname{Prob}(|\operatorname{vol}(S)-\operatorname{Vol}(S)|>\lambda)<e^{-\lambda^{2} /(2 \operatorname{Vol}(S)+\lambda / 3)} .
$$

For $k \geq 2$, we define the $k$-th moment of the expected volume by $\operatorname{Vol}_{k}(S)=$ $\sum_{v_{i} \in S} w_{i}^{k}$ and we write $\operatorname{Vol}_{k}(G)=\sum_{i} w_{i}^{k}$. In a graph $G$, the distance $d(u, v)$ between two vertices $u$ and $v$ is just the length of a shortest path joining $u$ and $v$ (if it exists). In a connected graph $G$, the average distance of $G$ is the average over all distances $d(u, v)$ for $u$ and $v$ in $G$. We consider very sparse graphs that are often not connected. If $G$ is not connected, we define the average distance to be the average among all distances $d(u, v)$ for pairs of $u$ and $v$ both belonging to the same connected component. The diameter of $G$ is the maximum distance $d(u, v)$, where $u$ and $v$ are in the same connected component. Clearly, the diameter is 
at least as large as the average distance. All our graphs typically have a unique large connected component, call the giant component, which contains a positive fraction of edges.

The expected degree sequence $\mathbf{w}$ for a graph $G$ on $n$ vertices in $G(\mathbf{w})$ is said to be strongly sparse if we have the following:

(i) The second order average degree $\tilde{d}$ satisfies $0<\log \tilde{d} \ll \log n$.

(ii) For some constant $c>0$, all but $o(n)$ vertices have expected degree $w_{i}$ satisfying $w_{i} \geq c$. The average expected degree $d=\sum_{i} w_{i} / n$ is strictly greater than 1, i.e., $d>1+\epsilon$ for some positive value $\epsilon$ independent of $n$.

The expected degree sequence $\mathbf{w}$ for a graph $G$ on $n$ vertices in $G(\mathbf{w})$ is said to be admissible if the following condition holds, in addition to the assumption that $\mathbf{w}$ is strongly sparse.

(iii) There is a subset $U$ satisfying:

$$
\operatorname{Vol}_{2}(U)=(1+o(1)) \operatorname{Vol}_{2}(G) \gg \frac{\operatorname{Vol}_{3}(U) \log \tilde{d} \log \log n}{\tilde{d} \log n} .
$$

The expected degree sequence $\mathbf{w}$ for a graph $G$ on $n$ vertices is said to be specially admissible if (i) is replaced by (i') and (iii) is replaced by (iii'):

(i') $\log \tilde{d}=O(\log d)$.

(iii') There is a subset $U$ satisfying

$$
\operatorname{Vol}_{3}(U)=O\left(\operatorname{Vol}_{2}(G)\right) \frac{\tilde{d}}{\log \tilde{d}}, \quad \operatorname{andVol}_{2}(U)>d \operatorname{Vol}_{2}(G) / \tilde{d} .
$$

In this paper, we will prove the following:

Theorem 2.I. For a random graph $G$ with admissible expected degree sequence $\left(w_{1}, \ldots, w_{n}\right)$, the average distance is almost surely $(1+o(1)) \frac{\log n}{\log \tilde{d}}$.

Corollary 2.2. If $n p \geq c>1$ for some constant $c$, then almost surely the average distance of $G(n, p)$ is $(1+o(1)) \frac{\log n}{\log n p}$, provided $\frac{\log n}{\log n p}$ goes to infinity as $n \rightarrow \infty$.

The proof of Corollary 2.2 follows by taking $w_{i}=n p$ and $U$ to be the set of all vertices. It is easy to verify in this case that $\mathbf{w}$ is admissible, so Theorem 2.1 applies. 
Theorem 2.3. For a random graph $G$ with a specially admissible degree sequence $\left(w_{1}, \ldots, w_{n}\right)$, the diameter is almost surely $\Theta(\log n / \log \tilde{d})$.

Corollary 2.4. If $n p=c>1$ for some constant $c$, then almost surely the diameter of $G(n, p)$ is $\Theta(\log n)$.

Theorem 2.5. For a power law random graph with exponent $\beta>3$ and average degree $d$ strictly greater than 1 , almost surely the average distance is $(1+o(1)) \frac{\log n}{\log \tilde{d}}$ and the diameter is $\Theta(\log n)$.

Theorem 2.6. Suppose a power law random graph with exponent $\beta$ has average degree $d$ strictly greater than 1 and maximum degree $m$ satisfying $\log m \gg$ $\log n / \log \log n$. If $2<\beta<3$, almost surely the diameter is $\Theta(\log n)$ and the average distance is at most $(2+o(1)) \frac{\log \log n}{\log (1 /(\beta-2))}$.

For the case of $\beta=3$, the power law random graph has diameter almost surely $\Theta(\log n)$ and has average distance $\Theta(\log n / \log \log n)$.

\section{Neighborhood Expansion and Connected Components}

Here, we state several useful facts concerning the distances and neighborhood expansions in $G(\mathbf{w})$. These facts are not only useful for the proofs of the main theorems but also are of interest on their own right. The proofs can be found in [Chung and Lu 01, Chung and Lu 03]

Lemma 3.I. In a random graph $G$ in $G(\mathbf{w})$ with a given expected degree sequence $\mathbf{w}=\left(w_{1}, \ldots, w_{n}\right)$, for any fixed pairs of vertices $(u, v)$, the distance $d(u, v)$ between $u$ and $v$ is greater than $\left\lfloor\frac{\log \operatorname{Vol}(G)-c}{\log \tilde{d}}\right\rfloor$ with probability at least $1-\frac{w_{u} w_{v}}{\tilde{d}(\tilde{d}-1)} e^{-c}$.

Lemma 3.2. In a random graph $G \in G(\mathbf{w})$, for any two subsets $S$ and $T$ of vertices, we have

$$
\operatorname{Vol}(\Gamma(S) \cap T) \geq(1-2 \epsilon) \operatorname{Vol}(S) \frac{\operatorname{Vol}_{2}(T)}{\operatorname{Vol}(G)}
$$

with probability at least $1-e^{-c}$ where $\Gamma(S)=\{v: v \sim u \in S$ and $v \notin S\}$, provided $\operatorname{Vol}(S)$ satisfies

$$
\frac{2 c \operatorname{Vol}_{3}(T) \operatorname{Vol}(G)}{\epsilon^{2} \operatorname{Vol}_{2}^{2}(T)} \leq \operatorname{Vol}(S) \leq \frac{\epsilon \operatorname{Vol}_{2}(T) \operatorname{Vol}(G)}{\operatorname{Vol}_{3}(T)}
$$


Lemma 3.3. For any two disjoint subsets $S$ and $T$ with $\operatorname{Vol}(S) \operatorname{Vol}(T)>c \operatorname{Vol}(G)$, we have

$$
\operatorname{Pr}(d(S, T)>1)<e^{-c}
$$

where $d(S, T)$ denotes the distance between $S$ and $T$.

Lemma 3.4. Suppose that $G$ is a random graph on $n$ vertices so that for a fixed value $c, G$ has o $(n)$ vertices of degree less than $c$, and has average degree $d$ strictly greater than 1. Then for any fixed vertex $v$ in the giant component, if $\tau=o(\sqrt{n})$, then there is an index $i_{0} \leq c_{0} \tau$ so that with probability at least $1-\frac{c_{1} \tau^{3 / 2}}{e^{c_{2} \tau}}$, we have

$$
\operatorname{Vol}\left(\Gamma_{i_{0}}(v)\right) \geq \tau
$$

where the $c_{i}$ are constants depending only on $c$ and $d$, while $\Gamma_{i}(S)=\Gamma\left(\Gamma_{i-1}(S)\right)$ for $i>1$ and $\Gamma_{1}(S)=\Gamma(S)$.

We remark that in the proofs of Theorem 2.1 and Theorem 2.3, we will take $\tau$ to be of order $\frac{\log n}{\log \tilde{d}}$. The statement of Lemma 3.4 is, in fact, stronger than what we will actually need.

Another useful tool is the following result in [Chung and Lu 03] on the expected sizes of connected components in random graphs with given expected degree sequences.

Lemma 3.5. Suppose that $G$ is a random graph in $G(\mathbf{w})$ with given expected degree sequence $\mathbf{w}$. If the expected average degree $d$ is strictly greater than 1 , then the following holds:

(1) Almost surely $G$ has a unique giant component. Furthermore, the volume of the giant component is at least $\left(1-\frac{2}{\sqrt{d e}}+o(1)\right) \operatorname{Vol}(G)$ if $d \geq \frac{4}{e}=1.4715 \ldots$, and is at least $\left(1-\frac{1+\log d}{d}+o(1)\right) \operatorname{Vol}(G)$ if $d<2$.

(2) The second largest component almost surely has size $O\left(\frac{\log n}{\log d}\right)$.

Proof of Theorem 2.I. Suppose $G$ is a random graph with an admissible expected degree sequence. From Lemma 3.5, we know that with high probability the giant component has volume at least $\Theta(\operatorname{Vol}(G))$. From Lemma 3.5, the sizes of all small components are $O(\log n)$. Thus, the average distance is primarily determined by pairs of vertices in the giant component.

From the admissibility condition (i), $\tilde{d} \leq n^{\epsilon}$ implies that only $o(n)$ vertices can have expected degrees greater than $n^{\epsilon}$. Hence, we can apply Lemma 3.1 (by choosing $c=3 \epsilon \log n$, for any fixed $\epsilon>0$ ) so that with probability $1-o(1)$, the 
distance $d(u, v)$ between $u$ and $v$ satisfies $d(u, v) \geq(1-3 \epsilon-o(1)) \log n / \log \tilde{d}$. Here, we use the fact that $\log \operatorname{Vol}(G)=\log \tilde{d}+\log n=(1+o(1)) \log n$. Because the choice of $\epsilon$ is arbitrary, we conclude the average distance of $G$ is almost surely at least $(1+o(1)) \log n / \log \tilde{d}$.

Next, we wish to establish the lower bound $(1+o(1)) \frac{\log \operatorname{Vol}(G)}{\log \tilde{d}}$ for the average distance between two vertices $u$ and $v$ in the giant component.

For any vertex $u$ in the giant component, we use Lemma 3.4 to see that for $i_{0} \leq C \epsilon \frac{\log n}{\log \tilde{d}}$, the $i_{0}$-boundary $\Gamma_{i_{0}}(v)$ of $v$ satisfies

$$
\operatorname{Vol}\left(\Gamma_{i_{0}}(v)\right) \geq \epsilon \frac{\log n}{\log \tilde{d}}
$$

with probability $1-o(1)$.

Next, we use Lemma 3.2 to deduce that $\operatorname{Vol}\left(\Gamma_{i}(u)\right)$ will grow roughly by a factor of $(1-2 \epsilon) \tilde{d}$ as long as $\operatorname{Vol}\left(\Gamma_{i}(u)\right)$ is no more than $\sqrt{c \operatorname{Vol}(G)}$ (by choosing $c=2 \log \log n)$. The failure probability is at most $e^{-c}$ at each step. Hence, for $i_{1} \leq \frac{\log (c \operatorname{Vol}(G))}{2 \log (1-2 \epsilon) \dot{d}}$ more steps, we have $\operatorname{Vol}\left(\Gamma_{i_{0}+i_{1}}(v)\right) \geq \sqrt{c \operatorname{Vol}(G)}$ with probability at least $1-i_{1} e^{-c}=1-o(1)$. Here, $i_{0}+i_{1}=(1+o(1)) \frac{\log n}{2 \log d}$. Similarly, for the vertex $v$, there are integers $i_{0}^{\prime}$ and $i_{1}^{\prime}$ satisfying $i_{0}^{\prime}+i_{1}^{\prime}=(1+o(1)) \frac{\log n}{2 \log \tilde{d}}$ so that $\operatorname{Vol}\left(\Gamma_{i_{0}^{\prime}+i_{1}^{\prime}}(v)\right) \geq \sqrt{c \operatorname{Vol}(G)}$ holds with probability at least $1-o(1)$.

By Lemma 3.3, with probability $1-o(1)$ there is a path connecting $u$ and $v$ with length $i_{0}+i_{1}+1+i_{0}^{\prime}+i_{1}^{\prime}=(1+o(1)) \frac{\log n}{\log \tilde{d}}$. Hence, almost surely the average distance of a random graph with an admissible degree sequence is $(1+o(1)) \frac{\log n}{\log \tilde{d}}$.

The proof of Theorem 2.3 is similar to that of Theorem 2.1 except that the special admissibility condition allows us to deduce the desired bounds with probability $1-o\left(n^{-2}\right)$. Thus, almost surely every pair of vertices in the giant components have mutual distance $O(\log n / \log \tilde{d})$.

\section{Random Power Law Graphs}

For random graphs with given expected degree sequences satisfying a power law distribution with exponent $\beta$, we may assume that the expected degrees are $w_{i}=c i^{-\frac{1}{\beta-1}}$ for $i$ satisfying $i_{0} \leq i<n+i_{0}$, as illustrated in Figures 1 and 2. Here, $c$ depends on the average degree and $i_{0}$ depends on the maximum degree $m$, namely, $c=\frac{\beta-2}{\beta-1} d n^{\frac{1}{\beta-1}}, i_{0}=n\left(\frac{d(\beta-2)}{m(\beta-1)}\right)^{\beta-1}$. 


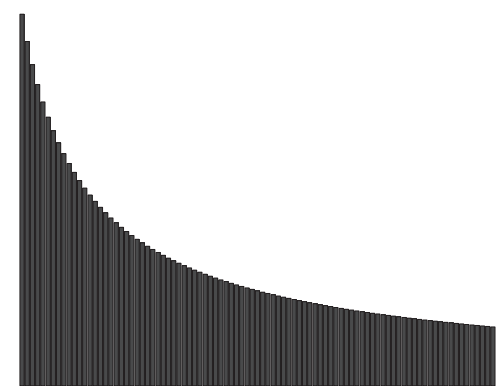

Figure I. Power law degree distribution.

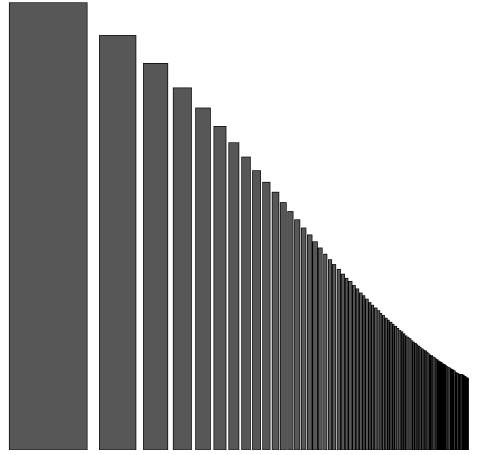

Figure 2. Log-scale of Figure 1.

The power law graphs with exponent $\beta>3$ are quite different from those with exponent $\beta<3$ as evidenced by the value of $\tilde{d}$ (assuming $m \gg d$ ).

$$
\tilde{d}= \begin{cases}(1+o(1)) d \frac{(\beta-2)^{2}}{(\beta-1)(\beta-3)} & \text { if } \beta>3 . \\ (1+o(1)) \frac{1}{2} d \ln \frac{2 m}{d} & \text { if } \beta=3 . \\ (1+o(1)) d^{\beta-2} \frac{(\beta-2)^{\beta-1} m^{3-\beta}}{(\beta-1)^{\beta-2}(3-\beta)} & \text { if } 2<\beta<3 .\end{cases}
$$

For the range of $\beta>3$, it can be shown that the power law graphs are both admissible and specially admissible. (One of the key ideas is to choose " $U$ " in condition (iii) or (iii') to be a set $U_{y}=\{v: \operatorname{deg}(v) \leq y\}$ for an appropriate $y$ independent of the maximum degree $m$. For example, choose $y$ to be $n^{1 / 4}$ for $\beta>4$, to be 4 for $\beta=4$ and to be $\log n /(\log d \log \log n)$ for $3<\beta<4)$. Theorem 2.5 then follows from Theorems 2.1 and 2.3 .

\section{I. The Range $2<\beta<3$}

Power law graphs with exponent $2<\beta<3$ have very interesting structures that can be roughly described as an "octopus" with a dense subgraph having small diameter as the core. We define $S_{k}$ to be the set of vertices with expected degree at least $k$. (We note that the set $S_{k}$ can be well approximated by the set of vertices with degree at least $k$.)

Here we outline the main ideas for the proof of Theorem 2.6.

Proof of Theorem 2.6. We define the core of a power law graph with exponent $\beta$ to be the set $S_{t}$ of vertices of degree at least $t=n^{1 / \log \log n}$.

Claim 4.I. The diameter of the core is almost surely $O(\log \log n)$. This follows from the fact that the core contains an Erdös-Renyi graph $G\left(n^{\prime}, p\right)$ with $n^{\prime}=c n t^{1-\beta}$ 
and $p=t^{2} / \operatorname{Vol}(G)$. From [Erdös and Rényi 59], this subgraph is almost surely connected. Using a result in [Chung and Lu 01], the diameter of this subgraph is, at most, $\frac{\log n^{\prime}}{\log p n^{\prime}}=(1+o(1)) \frac{\log n}{(3-\beta) \log t}=O(\log \log n)$.

Claim 4.2. Almost all vertices with degree at least $\log n$ are almost surely within distance $O(\log \log n)$ from the core. To see this, we start with a vertex $u_{0}$ with degree $k_{0} \geq \log ^{C} n$ for some constant $C=\frac{1.1}{(\beta-2)(3-\beta)}$. By applying Lemma 3.3, with probability at least $1-n^{-3}, u_{0}$ is a neighbor of some $u_{1}$ with degree $k_{1} \geq\left(k_{0} / \log ^{C} n\right)^{1 /(\beta-2)^{s}}$. We then repeat this process to find a path with vertices $u_{0}, u_{1}, \ldots, u_{s}$, and the degree $k_{s}$ of $u_{s}$ satisfies $k_{s} \geq\left(k_{0} / \log ^{C} n\right)^{1 /(\beta-2)^{s}}$ with probability $1-n^{-2}$. By choosing s to satisfy $\log k_{s} \geq \log n / \log \log n$, we are done.

Claim 4.3. For each vertex $v$ in the giant component, with probability $1-o(1), v$ is within distance $O(\log \log n)$ from a vertex of degree at least $\log ^{C} n$. This follows from Lemma 4 ( choosing $\tau=c \log \log \log n$ and the neighborhood expansion factor $\left.c^{\prime} \log \log \log n\right)$.

Claim 4.4. For each vertex $v$ in the giant component, with probability $1-o\left(n^{-2}\right), v$ is within distance $O(\log n)$ from a vertex of degree at least $O(\log n)$. Thus with probability $1-o(1)$, the diameter is $O(\log n)$.

The proofs of Claims 4.3 and 4.4 will be given in Section 5 .

Combining Claims 4.1-4.3, we have derived an upper bound $O(\log \log n)$ for the average distance. (By a similar but more careful analysis [Lu 02], this upper bound can be further improved to $c \log \log n$ for $c=\frac{2}{\log (1 /(\beta-2))}$.) From Claim 4.4, we have an upper bound $O(\log n)$ for the diameter.

Next, we will establish a lower bound of order $\log n$. We note that the minimum expected degree in a power law random graph with exponent $2<\beta<3$ as described in Section 4 is $(1+o(1)) \frac{d(\beta-2)}{\beta-1}$. We consider all vertices with expected degree less than the average degree $d$. By a straightforward computation, there are about $\left(\frac{\beta-2}{\beta-1}\right)^{\beta-1} n$ such vertices. For a vertex $u$ and a subset $T$ of vertices, the probability that $u$ has only one neighbor which has expected degree less than $d$ and is not adjacent to any vertex in $T$ is at least

$$
\begin{aligned}
& \sum_{w_{v}<d} w_{u} w_{v} \rho \prod_{j \neq v}\left(1-w_{u} w_{j} \rho\right) \\
& \approx w_{u} \operatorname{vol}\left(S_{d}\right) \rho e^{-w_{u}} \\
& \approx\left(1-\left(\frac{\beta-2}{\beta-1}\right)^{\beta-2}\right) w_{u} e^{-w_{u}}
\end{aligned}
$$


Note that this probability is bounded away from 0, (say, it is greater than $c$ for some constant $c$ ). Then, with probability at least $n^{-1 / 100}$, we have an induced path of length at least $\frac{\log n}{100 \log c}$ in $G$. Starting from any vertex $u$, we search for a path as an induced subgraph of length at least $\frac{\log n}{100 \log c}$ in $G$. If we fail to find such a path, we simply repeat the process by choosing another vertex as the starting point. Since $S_{d}$ has at least $\left(\frac{\beta-2}{\beta-1}\right)^{\beta-1} n$ vertices, then with high probability, we can find such a path. Hence, the diameter is almost surely $\Theta(\log n)$.

For the case of $\beta=3$, the power law random graph almost surely has diameter of order $\log n$, but the average distance is $\Theta(\log n / \log \tilde{d})=\Theta(\log n / \log \log n)$. The proof will be given in Section 5 .

\section{The Proofs}

This section contains proofs for Lemmas 3.1, 3.2, and 3.2 and Theorems 2.5 and 2.6.

Proof of Lemma 3.I. We choose $k=\left\lfloor\frac{\log \operatorname{Vol}(G)-c}{\log \tilde{d}}\right\rfloor$, satisfying

$$
(\tilde{d})^{k} \leq \operatorname{Vol}(G) e^{-c}
$$

For each fixed sequence of vertices, $\pi=\left(u=v_{0}, v_{1} \ldots v_{j-1}, v_{j}=v\right)$, the probability that $\pi$ is not a path of $G$ is

$$
1-w_{u} w_{v} w_{i_{1}}^{2} \cdots w_{i_{j-1}}^{2} \rho^{j}
$$

where $\rho=1 / \operatorname{Vol}(G)$. For a given sequence $\pi$, " $\pi$ is not a path of $G_{\beta}$ " is a monotone decreasing graph property. By the FKG inequality (see [Alon and Spencer 92]), we have

$$
\begin{aligned}
\operatorname{Pr}(d(u, v) \geq k) & \geq \prod_{j=1}^{k-1} \prod_{i_{1} \ldots i_{j-1}}\left(1-w_{u} w_{v} w_{i_{1}}^{2} \cdots w_{i_{j-1}}^{2} \rho^{j}\right) \\
& \approx \prod_{j=1}^{k-1} e^{-w_{u} w_{v} \rho^{j} \sum_{w_{1}, \ldots, w_{j-1}} w_{1}^{2} \cdots w_{j-1}^{2}} \\
& \approx e^{-w_{u} w_{v} \sum_{j=1}^{k-1} \rho^{j}\left(\sum_{i=1}^{n} w_{i}^{2}\right)^{j-1}} \\
& \approx e^{-w_{u} w_{v} \rho\left(\left(\sum_{i} w_{i}^{2} \rho\right)^{k-1}-1\right) /\left(\sum_{i} w_{i}^{2} \rho-1\right)} \\
& \geq e^{-w_{u} w_{v} e^{-c} / \tilde{d}(\tilde{d}-1)} \\
& \geq 1-\frac{w_{u} w_{v}}{\tilde{d}(\tilde{d}-1)} e^{-c}
\end{aligned}
$$

by the definition of $k$. 
We will use the following general inequality of large deviation [Chung and Lu 03] for the proof of Lemma 3.2.

Lemma 5.I. [Lu 02] Let $X_{1}, \ldots, X_{n}$ be independent random variables with

$$
\operatorname{Pr}\left(X_{i}=1\right)=p_{i}, \quad \operatorname{Pr}\left(X_{i}=0\right)=1-p_{i}
$$

For $X=\sum_{i=1}^{n} a_{i} X_{i}$, we have $E(X)=\sum_{i=1}^{n} a_{i} p_{i}$ and we define $\nu=\sum_{i=1}^{n} a_{i}^{2} p_{i}$. Then we have

$$
\operatorname{Pr}(X<E(X)-\lambda) \leq e^{-\lambda^{2} / 2 \nu}
$$

Proof of Lemma 3.2. Let $X_{j}$ be the indicated random variable that a vertex $v_{j} \in T$ is in $\Gamma(S)$. We have

$$
\begin{aligned}
\operatorname{Pr}\left(X_{j}=1\right) & =1-\prod_{v_{i} \in S}\left(1-w_{i} w_{j} \rho\right) \\
& \geq \operatorname{Vol}(S) w_{j} \rho-\operatorname{Vol}(S)^{2} w_{j}^{2} \rho^{2} .
\end{aligned}
$$

The volume of $\Gamma(S) \cap T$ is just $\sum_{v_{j} \in T} w_{j} X_{j}$. The expected value of $\operatorname{Vol}(\Gamma(S) \cap$ $T)$ is $\operatorname{Vol}(S) \operatorname{Vol}_{2}(T) \rho-\operatorname{Vol}(S)^{2} \operatorname{Vol}_{3}(T) \rho^{2}$. Using the inequality of large deviation in Lemma 5.1, with probability at least $1-e^{-c}$, we have

$$
\begin{aligned}
\operatorname{Vol}(\Gamma(S) \cap T) & =\sum_{v_{j} \in T} w_{j} X_{j} \\
& \geq \operatorname{Vol}(S) \operatorname{Vol}_{2}(T) \rho-\operatorname{Vol}(S)^{2} \operatorname{Vol}_{3}(T) \rho^{2}-\sqrt{2 c \operatorname{Vol}(S) \operatorname{Vol}_{3}(T) \rho} \\
& \geq(1-2 \epsilon) \operatorname{Vol}(S) \operatorname{Vol}_{2}(T) \rho
\end{aligned}
$$

by the assumption (3.1).

Proof of Lemma 3.3. For vertices $v_{i} \in S$ and $v_{j} \in T$, the probability that $v_{i} v_{j}$ is not an edge is

$$
1-w_{i} w_{j} \rho
$$

Since edges are independently chosen, we have

$$
\begin{aligned}
\operatorname{Pr}(d(S, T)>1) & =\prod_{v_{i} \in S, v_{j} \in T}\left(1-w_{i} w_{j} \rho\right) \\
& \leq e^{-\operatorname{Vol}(S) \operatorname{Vol}(T) \rho} \\
& <e^{-c} .
\end{aligned}
$$


Proof of Theorem 2.5. To show that a random power law graph with exponent $\beta$ is admissible or specially admissible, the key idea is to prove condition (iii) by choosing appropriate $U_{\mathrm{s}}$ for (iii) or (iii') while other conditions are easy to verify.

Let $U_{y}$ consist of all vertices with weight less than or equal to $d \frac{\beta-2}{\beta-1} y$ where $d$ is the average degree. We have

$$
\begin{gathered}
\operatorname{Vol}_{2}\left(U_{y}\right)=\sum_{i=\left\lceil n y^{-1 /(\beta-1)}\right\rceil}^{n} w_{i}^{2} \\
=d^{2} \frac{(\beta-2)^{2}}{(\beta-1)(\beta-3)} n\left(1-y^{-(\beta-3)}+O\left(\frac{y^{2}}{n}\right)\right) . \\
\operatorname{Vol}_{3}\left(U_{y}\right)=\sum_{i=\left\lceil n y^{-1 /(\beta-1)}\right\rceil}^{n} w_{i}^{3} \\
= \begin{cases}d^{3} \frac{(\beta-2)^{3}}{(\beta-1)^{2}(\beta-4)} n\left(1-y^{-(\beta-4)}+O\left(\frac{y^{3}}{n}\right)\right) & \text { if } \beta>4 . \\
\frac{8}{27} d^{3} n\left(\ln y+O\left(\frac{y^{3}}{n}\right)\right) . & \text { if } \beta=4 . \\
d^{3} \frac{(\beta-2)^{3}}{(\beta-1)^{2}(4-\beta)} n\left(y^{4-\beta}+O\left(\frac{y^{3}}{n}\right)\right) & \text { if } 3<\beta<4 .\end{cases}
\end{gathered}
$$

We consider the following three cases:

Case I: $\beta>4$. By choosing $y=n^{1 / 4}, U=U_{y}$ satisfies

$$
\begin{gathered}
\operatorname{Vol}_{2}(U)=d^{2} \frac{(\beta-2)^{2}}{(\beta-1)(\beta-3)} n(1+o(1))=(1+o(1)) \operatorname{Vol}_{2}(G), \\
\frac{\operatorname{Vol}_{3}(U)}{\operatorname{Vol}_{2}(U)}=(1+o(1)) d \frac{(\beta-1)(\beta-3)}{9}=O\left(\frac{\tilde{d}}{\log \tilde{d}}\right) .
\end{gathered}
$$

Thus the power law degree sequence with $\beta>4$ is both admissible and specially admissible.

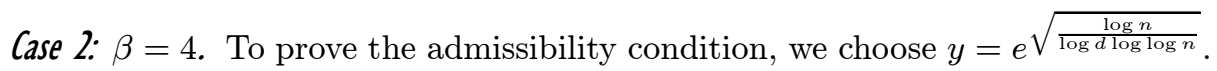
Then $U=U_{y}$ satisfies

$$
\begin{aligned}
& \operatorname{Vol}_{2}(U)=d^{2} \frac{(\beta-2)^{2}}{(\beta-1)(\beta-3)} n(1+o(1)) \\
&=(1+o(1)) \operatorname{Vol}_{2}(G), \\
& \frac{\mathrm{Vol}_{3}(U)}{\operatorname{Vol}_{2}(U)}=(1+o(1)) d \frac{2}{9} \log y=o\left(\frac{\tilde{d}}{\log \tilde{d}} \frac{\log n}{\log \log n}\right) .
\end{aligned}
$$

Hence, the power law degree sequence with $\beta=4$ is admissible. 
To prove the specially admissibility condition, we choose $y=4$. Then $U=U_{y}$ satisfies

$$
\begin{aligned}
\operatorname{Vol}_{2}(U) & =d^{2} \frac{(\beta-2)^{2}}{(\beta-1)(\beta-3)} n\left(1-\frac{1}{4}+o(1)\right) \\
= & \left(\frac{3}{4}+o(1)\right) \operatorname{Vol}_{2}(G) \\
\approx & d \operatorname{Vol}_{1}(G), \\
\frac{\operatorname{Vol}_{3}(U)}{\operatorname{Vol}_{2}(U)} & =(1+o(1)) d \frac{8}{27} d \log 4 \\
& =O\left(\frac{\tilde{d}}{\log \tilde{d}}\right),
\end{aligned}
$$

since the average degree $d$ is bounded above by a constant in this case. Thus, the power law degree sequence with $\beta=4$ is specially admissible.

Case 3: $3<\beta<4$. To prove admissibility, we choose $y=\frac{\log n}{\log d \log \log n}$. Then, $U=U_{y}$ satisfies

$$
\begin{aligned}
\operatorname{Vol}_{2}(U)=d^{2} & \frac{(\beta-2)^{2}+o(1)}{(\beta-1)(\beta-3)} n=(1+o(1)) \operatorname{Vol}_{2}(G), \\
\frac{\operatorname{Vol}_{3}(U)}{\operatorname{Vol}_{2}(U)} & =(1+o(1)) d \frac{(\beta-2)(\beta-3)}{(\beta-1)(4-\beta)} y^{1 /(4-\beta))} \\
& =o\left(\frac{\tilde{d}}{\log \tilde{d}} \frac{\log n}{\log \log n}\right) .
\end{aligned}
$$

Hence, the power law degree sequence with $3<\beta<4$ is admissible.

To prove the specially admissibility condition, we choose $y=(\beta-2)^{\frac{2}{\beta-3}}$. Then $U=U_{y}$ satisfies

$$
\begin{aligned}
\operatorname{Vol}_{2}(U)= & d^{2} \frac{(\beta-2)^{2}}{(\beta-1)(\beta-3)} n\left(1-\frac{1}{(\beta-2)^{2}}+o(1)\right) \\
= & (d+o(1)) \operatorname{Vol}(G), \\
\frac{\mathrm{Vol}_{3}(U)}{\operatorname{Vol}_{2}(U)} & =(1+o(1)) d \frac{(\beta-2)(\beta-3)}{(\beta-1)(4-\beta)} y^{1 /(4-\beta)} \\
= & O\left(\frac{\tilde{d}}{\log \tilde{d}}\right) .
\end{aligned}
$$

Hence, the power law degree sequence with $3<\beta<4$ is specially admissible and the proof is complete. 
Proof of Claim 4.3. The main tools are Lemma 3.2 and Lemma 3.4. To apply Lemaa 3.4, we note that the minimum expected degree (weight) is $w_{\min }=(1+$ $o(1)) \frac{d(\beta-2)}{\beta-1}$ and $d>1$. We want to show that some $i$-neighborhood of $u$ will grow "large" enough to apply Lemma 3.2. Let $S$ be $i$-th neighborhood of $u$, consisting of all vertices within distance $i$ from $u$. Let $T=S\left(w_{\min }, a\right)$ denote the set of vertices with weights between $w_{\min }$ and $a w_{\min }$. Here, $a$ is some large value to be chosen later. We have

$$
\begin{aligned}
\operatorname{Vol}(T) & \approx n d\left(1-a^{2-\beta}\right) \\
\operatorname{Vol}_{2}(T) & \approx n d^{2}\left(1-\frac{1}{\beta-1}\right)^{2} \frac{\beta-1}{3-\beta} a^{3-\beta} \\
\operatorname{Vol}_{3}(T) & \approx n d^{3}\left(1-\frac{1}{\beta-1}\right)^{3} \frac{\beta-1}{4-\beta} a^{4-\beta} .
\end{aligned}
$$

To apply Lemma $3.2, \operatorname{Vol}(\Gamma(S))$ must satisfy:

$$
\begin{aligned}
\operatorname{Vol}(\Gamma(S)) & \geq \frac{2 c}{\epsilon^{2}} \frac{\operatorname{Vol}_{3}(T)}{\operatorname{Vol}_{2}^{2}(T)} \operatorname{Vol}(G) \\
& \approx \frac{2 c}{\epsilon^{2}} \frac{(3-\beta)^{2}}{(\beta-2)(4-\beta)} a^{\beta-2}
\end{aligned}
$$

and

$$
\begin{aligned}
\operatorname{Vol}(\Gamma(S)) & \leq \epsilon \frac{\operatorname{Vol}_{2}(T)}{\operatorname{Vol}_{3}(T)} \operatorname{Vol}(G) \\
& \approx \epsilon \frac{(\beta-2)(3-\beta)}{(\beta-1)(4-\beta) a} n .
\end{aligned}
$$

Both the above equations are easy to satisfy by appropriately choosing the values for "c" and " $\epsilon$." For example, we can select " $a$ " = " $c$ " = $\log \log \log n, " \epsilon "=\frac{1}{4}$, and " $\tau$ " $=\log \log \log n$. Then, Lemma 3.4 implies that there are constants $c_{0}, c_{1}, c_{2}$ and an index $i_{0} \leq c_{0} \tau$ so that we have

$$
\operatorname{Vol}\left(\Gamma_{i_{0}}(u)\right) \geq \tau
$$

with probability at least $1-\frac{c_{1} \tau^{3 / 2}}{e^{c_{2} \tau}}=1-o(1)$. By Lemma 3.2 , with probability at least $1-e^{-c}=1-\frac{1}{\log \log n}$, the volume of $\Gamma_{i}(u)$ for $i>i_{0}$ will grow at a rate greater than

$$
(1-2 \epsilon) \frac{\operatorname{Vol}_{2}(T)}{\operatorname{Vol}(G)} \approx \frac{\left.d(\beta-2)^{2}\right)}{2(\beta-1)(3-\beta)} a^{3-\beta},
$$

if $\Gamma_{i}(u)$ has volume not too large $(<\sqrt{n})$. After, at most, $(1+o(1)) \frac{2 \log \log n}{(3-\beta) \log a}=$ $o(\log \log n)$ steps, the volume of the reachable vertices is at least $\log ^{2} n$. Lemma 
3.3 then implies that with one additional step, we can reach a vertex of weight $\log ^{C} n$ with probablility at least $1-e^{-\log ^{2} n}$ The total number of steps is, at most,

$$
c_{0} \tau+o(\log \log n)+1=o(\log \log n) .
$$

The total failure probability for $u$ to reach a vertex of weight at least $\log ^{C} n$ is, at most,

$$
o(1)+o(\log \log n) \frac{1}{\log \log n}+e^{-\Theta\left(\log ^{2} n\right)}=o(1) .
$$

Claim 4.3 is proved.

Proof of Claim 4.4. To prove that the diameter is $O(\log n)$ with probability $1-o(1)$, it suffices to show that for each vertex $v$ in the giant component, with probability $1-o\left(n^{-2}\right), v$ is within distance $O(\log n)$ from a vertex of degree at least $O(\log n)$. To apply Lemma 3.4 , we choose " $a "=100, " c "=3 \log n$, " $\epsilon "=\frac{1}{4}$, and " $\tau$ " = $\left(\frac{3}{c_{2}}+96\right) \frac{(\beta-3)^{3}}{(\beta-2)(4-\beta)} 100^{\beta-2} \log n$. Similar to the proof for Claim 4.3 , the total failure probability for $u$ to reach a vertex of weight at least $\log ^{C} n$ is, at most,

$$
\frac{c_{0} \tau^{3 / 2}}{e^{c_{2} \tau}}+O(\log \log n) e^{-3 \log n}+e^{-\Theta\left(\log ^{2} n\right)}=o\left(\frac{1}{n^{2}}\right) .
$$

The total number of steps is, at most,

$$
c_{0} \tau+O(\log \log n)+1=O(\log n) .
$$

Now we will show a lower bound of $\Theta(\log n)$ for the diameter. Recall that the minimum weight is $w_{\min }=\frac{d(\beta-2)}{\beta-1}$. We consider all vertices with weight less than $d$. There are $\left(\frac{\beta-2}{\beta-1}\right)^{\beta-1} n$ such vertices. For a vertex $u$, the probability that $u$ has only one neighbor and having weight less than $d$ is at least

$$
\begin{aligned}
& \sum_{w_{v}<d} w_{u} w_{v} \rho \prod_{j \neq v}\left(1-w_{u} w_{j} \rho\right) \\
& \approx w_{u} \operatorname{Vol}\left(S\left(w_{\min }, \frac{\beta-1}{\beta-2}\right)\right) \rho e^{-w_{u}} \\
& \approx\left(1-\left(\frac{\beta-2}{\beta-1}\right)^{\beta-2}\right) w_{u} e^{-w_{u}} .
\end{aligned}
$$

Note that this probability is larger than some constant $c$. Thus with probability at least $n^{-1 / 100}$, we have an induced path of length $\frac{\log n}{100 \log c}$. Starting with a vertex $u$, we search for a path of length $\frac{\log n}{100 \log c}$ as an induced subgraph in $S\left(w_{\min }, \frac{\beta-1}{\beta-2}\right)$. If we fail to find such a path, we simply repeat the process by selecting another starting vertex. Since $S\left(w_{\min }, \frac{\beta-1}{\beta-2}\right)$ has $\left(\frac{\beta-2}{\beta-1}\right)^{\beta-1} n$ vertices, with high probability, we will find such a path. Hence, the diameter is $\Theta(\log n)$. 
Proof of Theorem 2.6 for the case $\beta=3$. We first examine the following. Let $T$ denote the set of vertices with weights less than $t$. Then we have

$$
\begin{aligned}
& \operatorname{Vol}(T)=\sum_{i=n\left(\frac{d}{2 t}\right)^{2}}^{n} \frac{d}{2}\left(\frac{i}{n}\right)^{-1 / 2} \\
& \approx n \int_{\left(\frac{d}{2 t}\right)^{2}}^{n} \frac{d}{2} x^{-1 / 2} d x \\
& =n d\left(1-\frac{d}{2 t}\right) \text {, } \\
& \operatorname{Vol}_{2}(T)=\sum_{i=n\left(\frac{d}{2 t}\right)^{2}}^{n} \frac{d^{2}}{4}\left(\frac{i}{n}\right)^{-1} \\
& \approx n \int_{\left(\frac{d}{2 t}\right)^{2}}^{n} \frac{d^{2}}{4} x^{-1} d x \\
& =\frac{n d^{2}}{2} \log \frac{2 t}{d} \text {, } \\
& \operatorname{Vol}_{3}(T)=\sum_{i=n\left(\frac{d}{2 t}\right)^{2}}^{n} \frac{d^{3}}{8}\left(\frac{i}{n}\right)^{-3 / 2} \\
& \approx n \int_{\left(\frac{d}{2 t}\right)^{2}}^{n} \frac{d^{3}}{8} x^{-3 / 2} d x \\
& =\frac{n d^{3}}{4}\left(\frac{2 t}{d}-1\right) \\
& =\frac{n d^{2}}{2}\left(t-\frac{d}{2}\right) \text {. } \\
& \frac{2 c \operatorname{Vol}_{3}(T) \operatorname{Vol}(G)}{\epsilon^{2} \operatorname{Vol}_{2}^{2}(T)} \approx \frac{2 c \frac{n d^{2}}{2}\left(t-\frac{d}{2}\right) n d}{\epsilon^{2}\left(\frac{n d^{2}}{2} \log \frac{2 t}{d}\right)^{2}} \approx \frac{2 c(2 t / d-1)}{\epsilon^{2} \log ^{2} 2 t / d} .
\end{aligned}
$$

We state the following useful lemma which is an immediate consequence of Lemma 3.2.

Lemma 5.2. Suppose a random power law graph with exponent $\beta=3$ has average degree $d$. For any $\epsilon<1 / 2, c>0$, any set $S$ with supposed $\operatorname{Vol}(S)>\frac{2 c}{\epsilon^{2}} \frac{2 t / d}{\log ^{2} 2 t / d}$ 
and $\operatorname{Vol}(S) \leq n^{2 / 3}$, satisfies

$$
\operatorname{Vol}(\Gamma(S))>(1-2 \epsilon) \frac{d}{2} \log \frac{2 t}{d} \operatorname{Vol}(S)
$$

with probability at least $1-e^{-c}$.

By Lemma 3.1, almost surely the average distance is at least $(1+o(1)) \frac{\log n}{\log \tilde{d}}$. Now we will prove an upper bound by establishing a series of facts.

Claim 5.3. For a vertex $u$ in the giant component, with probability at least $1-\frac{1}{\log ^{2} n}$, the volume of $\Gamma_{i_{1}}(u)$ is at least $\log ^{6} \log n$. for some $i_{1}=O\left(\log ^{6} \log n\right)$.

Proof of Claim 5.3. We use Lemma 3.1, with the choice of " $\tau$ " $=\log ^{6} \log n$. Thus, with probability at least $1-\frac{1}{\log ^{2} n}$, there are a constant $C$ and an index $i_{0}$ satisfying: $i_{0} \leq C \log ^{6} \log n$ and $\operatorname{Vol}\left(\Gamma_{i_{0}}(u)\right) \geq \log ^{6} \log n$.

Claim 5.4. With probability at least $1-o\left(\frac{1}{\log ^{2} n}\right)$, a subset $S$ with $\operatorname{Vol}(S) \geq \log ^{6} \log n$ has $\operatorname{Vol}\left(\Gamma_{i}(S)\right)>m$ if $i>\frac{\log m}{\log \log m}$.

Proof of Claim 5.4. We apply Lemma 5.2 repeatedly. At each step, we choose "c" $=\log ^{2} \log n, " \epsilon "=\frac{1}{\log \log n}$, and " $t "=\frac{d \epsilon^{2} a_{i}}{4 c} \log ^{2} \frac{\epsilon^{2} a_{i}}{2 c}$ where $a_{i}$ is defined recursively as follows. First, we define $a_{0} \geq \log ^{6} \log n$. For $i \geq 1$, we define $a_{i+1}=\frac{d}{10} a_{i} \log a_{i}$. We note that $a_{i+1}>a_{i}$ and $a_{i} \geq \log ^{6} \log n$. We will prove by induction that $\operatorname{Vol}\left(\Gamma_{i}(S)\right) \geq a_{i}$. From Claim 5.3, it holds for $i=0$. Suppose that it is true for $i$. We verify the assumption for Lemma 5.2 since

$$
\begin{aligned}
\frac{2 t / d}{\log ^{2} 2 t / d} & =\frac{\epsilon^{2} a_{i}}{2 c} \frac{\log ^{2} \frac{\epsilon^{2} a_{i}}{2 c}}{\left(\log \frac{\epsilon^{2} a_{i}}{2 c}+2 \log \log \frac{\epsilon^{2} a_{i}}{2 c}\right)^{2}} \\
& \leq \frac{\epsilon^{2} a_{i}}{2 c} \\
& \leq \frac{\epsilon^{2} \operatorname{Vol}\left(\Gamma_{i}(S)\right)}{2 c} .
\end{aligned}
$$

Hence,

$$
\begin{aligned}
\operatorname{Vol}\left(\Gamma_{i+1}(S)\right) & \geq(1-2 \epsilon) a_{i} \frac{d}{2} \log \frac{2 t}{d} \\
& \geq \frac{d}{10} a_{i} \log a_{i} . \\
& =a_{i+1}
\end{aligned}
$$


Next we will inductively prove $a_{i} \geq(i+s)^{i+s}$ for $s=e^{10 e / d}$. We can assume that $a_{0}=\log ^{6} \log n \geq s^{s}$ since $s$ is bounded. For $i+1$, we have

$$
\begin{aligned}
a_{i+1} & \geq \frac{d}{10} a_{i} \log a_{i} \\
& \geq \frac{d}{10}(i+s)^{i+s}(i+s) \log (i+s) \\
& \approx(i+1+s)^{i+1+s} \frac{d}{10 e} \log (i+s) \\
& >(i+1+s)^{i+1+s} .
\end{aligned}
$$

Therefore, we have proved that $a_{i} \geq(i+s)^{i+s}$. Let $i=\frac{\log m}{\log \log m-\log \log \log m}-s=$ $(1+o(1)) \frac{\log m}{\log \log m}$. Then,

$$
\begin{aligned}
a_{i} & \geq(i+s)^{i+s} \\
& \geq m
\end{aligned}
$$

Claim 5.5. With probability at least $1-o\left(\frac{1}{\log ^{2} n}\right)$, a subset $S$ with $\operatorname{Vol}(S) \geq m$ satisfies $\operatorname{Vol}\left(\Gamma_{i}(S)\right)>\sqrt{n} \log n$ if $i>\frac{(1+o(1))(\log \sqrt{n}-\log m)}{\log \left(\frac{d}{2} \log m\right)}$.

Proof of Claim 5.5. To apply Lemma 5.2, we choose "c" $=\log ^{2} \log n$, " $\epsilon$ " $=\frac{1}{\log \log n}$, and " $t$ " $=m$. The assumptions of Lemma 5.2 can be easily verified as follows:

$$
\begin{aligned}
\operatorname{Vol}(S) & \geq m \\
& \geq \frac{2 c}{\epsilon^{2}} \frac{2 m / d}{\log 2 m / d}
\end{aligned}
$$

Here, we use the assumption $m>n^{\delta}$. With probability $1-O\left(\frac{1}{\log ^{2} \log n}\right)$, the volume $\Gamma_{i}(S)$ grows at the rate of $(1-2 \epsilon) \tilde{d}$ as $i$ increases. Claim 5.5 is proved.

By Lemma 3.3, almost surely the distance of two sets with weight greater than $\sqrt{n} \log n$ is at most 1. By Claims 4.1-4.3, almost surely the distance of $u$ and $v$ in the giant connected component is

$$
\begin{gathered}
2\left(O\left(\log ^{6} \log n\right)+(1+o(1)) \frac{\log m}{\log \log m}+\right. \\
\left.\frac{(1+o(1))(1 / 2 \log n-\log m)}{\log \left(\frac{d}{2} \log m\right)}\right)=\Theta\left(\frac{\log n}{\log \log m}\right) .
\end{gathered}
$$


To derive an upper bound for the diameter, we need the following:

Claim 5.6. For a vertex $u$ in the giant component, with probability at least $1-\frac{1}{n^{3}}$, the volume of $\Gamma_{i_{1}}(u)$ is at least $8 e^{4} \log n$ for some $i_{1}=O(\log n)$.

Proof of Claim 5.6. By choosing " $\tau$ " $=8 e^{4} \log n$, Lemma 3.1, implies that there are a constant $C$ and an index $i_{0}$ satisfying: $i_{0} \leq 8 C e^{4} \log n$ and $\operatorname{Vol}\left(\Gamma_{i_{0}}(u)\right) \geq$ $8 e^{4} \log n$ with probability at least $1-n^{-3}$.

Claim 5.7. With probability at least $1-n^{-3}$, any subset $S$ with $\operatorname{Vol}(S) \geq 8 e^{4} \log n$ satisfies $\operatorname{Vol}_{i}(S)>\sqrt{n} \log n$ if $i>(1+o(1)) \frac{\log n}{2 \log d}$.

Proof of Claim 5.7. We will apply Lemma 5.2 with the choice of "c" $=4 \log n$, " $\epsilon "=\frac{1}{4}$ and " $t "=\frac{e^{4} d}{2}$. Note that

$$
\frac{2 c}{\epsilon^{2}} \frac{2 t / d}{\log ^{2} 2 t / d}=8 e^{4} \log n .
$$

By Lemma 5.2, with probability $1-n^{4}$ at each step, the volume of $i$-neighborhoods of $S$ grows at the rate of

$$
(1-2 \epsilon) \frac{d}{2} \log (2 t / d)=d
$$

if the volume of $\Gamma_{i}(S)$ is $O(\sqrt{n})$. By Claims 4.4 and 5.3, with probability at least $1-1 / n$, for all pair of vertices $u$ and $v$ in the giant component, the distance between $u$ and $v$ is at most

$$
2\left(O(\log n)+(1+o(1)) \frac{\log n}{2 \log d}\right)+1=O(\log n) .
$$

The lower bound $\Theta(\log n)$ of the diameter follows the same argument as in the proof for the range $2<\beta<3$.

The proof of Theorem 2.6 is complete.

\section{Summary}

When random graphs are used to model large complex graphs, the small world phenomenon of having short characteristic paths is well captured in the sense that with high probability, power law random graphs with exponent $\beta$ have average 


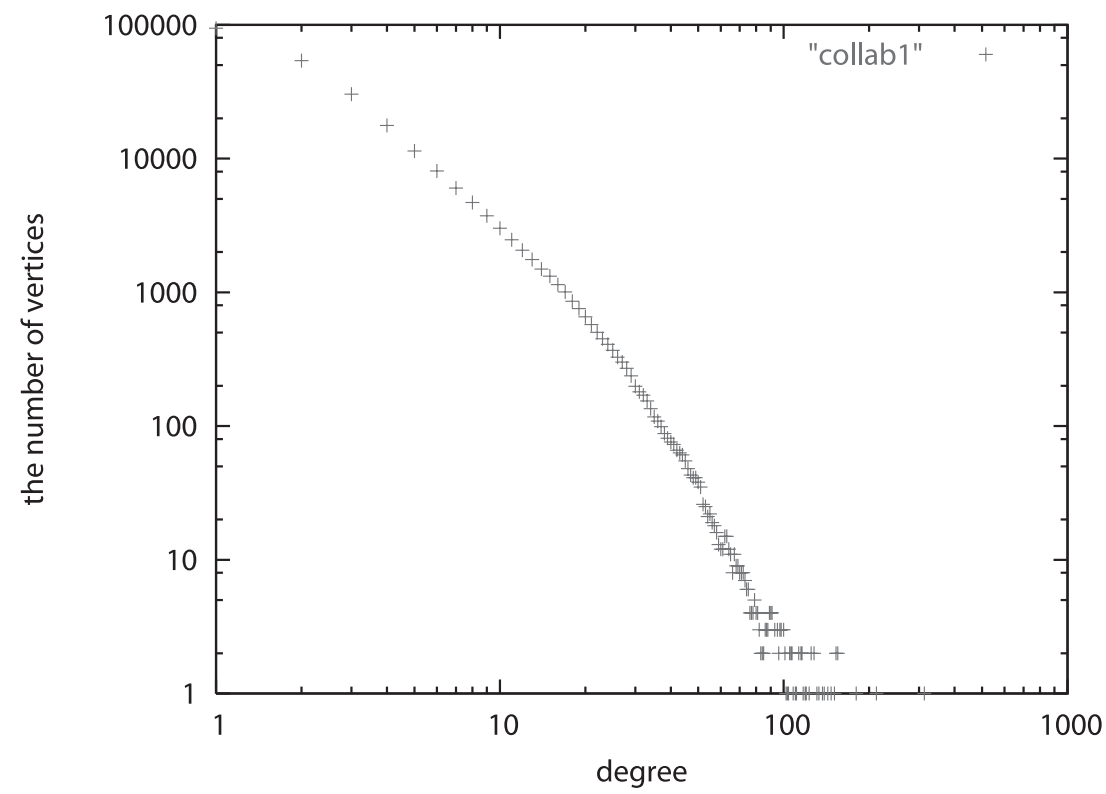

Figure 3. The power law degree distribution of the Collaboration Graph $G_{2}$.

distance of order $\log n$ if $\beta>3$, and of order $\log \log n$ if $2<\beta<3$. Thus, a phase transition occurs at $\beta=3$ and, in fact, the average distance of power law random graphs with exponent 3 is of order $\log n / \log \log n$. More specifically, for the range of $2<\beta<3$, there is a distinct core of diameter $\log \log n$ so that almost all vertices are within distance $\log \log n$ from the core, while almost surely there are vertices of distance $\log n$ away from the core.

Another aspect of the small world phenomenon concerns the so-called clustering effect, which asserts that two people who share a common friend are more likely to know each other. However, the clustering effect does not appear in random graphs and some explanation is in order. A typical large network can be regarded as a union of two major parts: a global network and a local network. Power law random graphs are suitable for modeling the global network while the clustering effect is part of the distinct characteristics of the local network.

Based on the data graciously provided by Jerry Grossman [Grossman et al. 03], we consider two types of collaboration graphs with roughly 337,000 authors as vertices. The first collaboration graph $G_{1}$ has about 496,000 edges with each edge joining two coauthors. It can be modeled by a random power law graph with exponent $\beta_{1}=2.97$ and $d=2.94$. The second collaboration graph $G_{2}$ has about 226,000 edges, each representing a joint paper with exactly two authors. The collaboration graph $G_{2}$ corresponds to a power law graph with exponent 


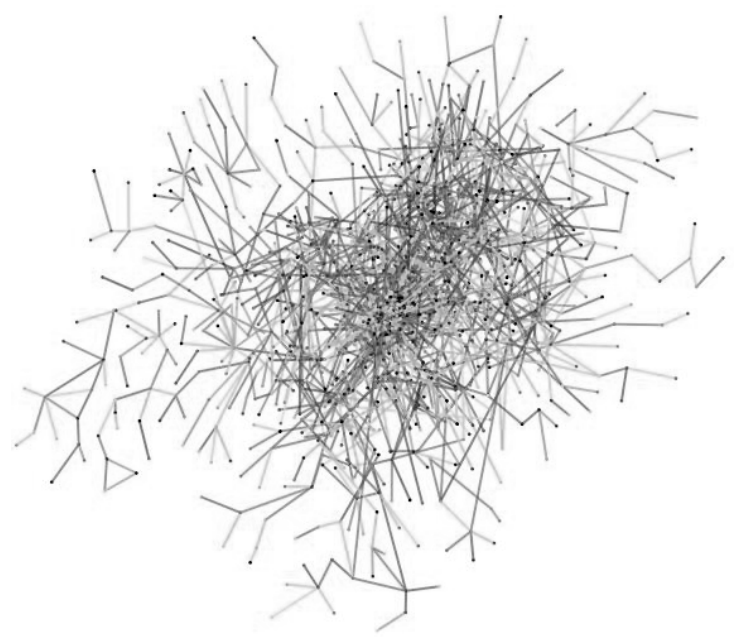

Figure 4. An induced subgraph of the collaboration graph $G_{2}$.

$\beta_{2}=3.26$ and $d=1.34$ (see Figures 3 and 4 ). Theorem 2.5 predicts that the value for the average distance in this case should be 9.89 (with a lower order error term). In fact, the actual average distance in this graph is 9.56 (see [Grossman et al. 03]).

Acknowledgments. This research was supported in part by NSF Grants DMS 0100472 and ITR 0205061. A short version of this paper (without complete proofs) has appeared in the Proceedings of the National Academy of Sciences.

\section{References}

[Aiello et al. 00] W. Aiello, F. Chung, and L. Lu. "A Random Graph Model for Massive Graphs." Proceedings of the Thirty-Second Annual ACM Symposium on Theory of Computing, pp. 171-180. New York: ACM Press, 2000.

[Aiello et al. 01a] W. Aiello, F. Chung, and L. Lu. "A Random Graph Model for Power Law Graphs." Experimental Math. 10 (2001), 53-66.

[Aiello et al. 01b] W. Aiello, F. Chung, and L. Lu. "Random Evolution in Massive Graphs." In Handbook of Massive Data Sets, Vol. 2, pp. 97-122. Dordrecht: Kluwer Academic Publishers, 2002. An extended abstract appeared in The 42th Annual Symposium on Foundation of Computer Sciences, pp. 510-519. Los Alamitos: IEEE Computer Society, 2001.

[Alon and Spencer 92] N. Alon and J. H. Spencer. The Probabilistic Method. New York: Wiley and Sons, 1992. 
[Albert et al. 99] R. Albert, H. Jeong, and A. Barabási. "Diameter of the World Wide Web." Nature 401 (1999), 130-131.

[Barabási and Albert 99] Albert-László Barabási and Réka Albert. "Emergence of Scaling in Random Networks. Science 286 (1999), 509-512.

[Broder et al. 00] A. Broder, R. Kumar, F. Maghoul, P. Raghavan, S. Rajagopalan, R. Stata, A. Tompkins, and J. Wiener. "Graph Structure in the Web." In Proceedings of the WWW9 Conference, pp. 309-320. Amsterdam: Elsevier Science, 2000.

[Chung and Lu 01] Fan Chung and Linyuan Lu. "The Diameter of Random Sparse Graphs." Advances in Applied Math. 26 (2001), 257-279.

[Chung and Lu 03] Fan Chung and Linyuan Lu. "Connected Components in a Random Graph with Given Degree Sequences." To appear in Annals of Combinatorics.

[Erdős and Gallai 59] P. Erdős and T. Gallai. "Gráfok előírt fokú pontokkal (Graphs with Points of Prescribed Degrees, in Hungarian)." Mat. Lapok 11 (1961), 264-274.

[Erdős and Rényi 59] P. Erdős and A. Rényi. "On Random Graphs I." Publ. Math. Debrecen 6 (1959), 290-291.

[Faloutsos et al. 99] M. Faloutsos, P. Faloutsos, and C. Faloutsos. "On Power-Law Relationships of the Internet Topology." In SIGCOMM 1999, pp. 251-262. New York: ACM Press, 1999.

[Grossman et al. 03] Jerry Grossman, Patrick Ion, and Rodrigo De Castro. "Facts about Erdős Numbers and the Collaboration Graph." Available from the World Wide Web: (http://www.oakland.edu/ grossman/trivia.html), 2003.

[Jeong et al. 00] H. Jeong, B. Tomber, R. Albert, Z. Oltvai, and A. L. Babárasi. "The Large-Scale Organization of Metabolic Networks." Nature 407 (2000), 378-382.

[Kleinberg et al. 99] J. Kleinberg, S. R. Kumar, P. Raphavan, S. Rajagopalan, and A. Tomkins. "The Web as a Graph: Measurements, Models and Methods." In Proceedings of the International Conference on Combinatorics and Computing, BerlinHeidelberg-New York: Springer-Verlag, 1999.

[Lotka 26] A. J. Lotka. "The Frequency Distribution of Scientific Productivity." The Journal of the Washington Academy of the Sciences 16 (1926), 317.

[Lu 01] Linyuan Lu. "The Diameter of Random Massive Graphs." In Proceedings of the Twelfth ACM-SIAM Symposium on Discrete Algorithms, pp. 912-921. New York: ACM Press, 2001.

[Lu 02] Linyuan Lu. "Probabilistic Methods in Massive Graphs and Internet Computing." Ph. D. diss., University of California, 2002. Available from the World Wide Web: (http://math.ucsd.edu/ llu/thesis.pdf), 2002.

[Milgram 67] S. Milgram. "The Small World Problem." Psychology Today 2 (1967), 60-67.

[Molloy and Reed 95] Michael Molloy and Bruce Reed. "A Critical Point for Random Graphs with a Given Degree Sequence." Random Structures and Algorithms 6:2-3 (1995), 161-179. 
[Molloy and Reed 98] Michael Molloy and Bruce Reed. "The Size of the Giant Component of a Random Graph with a Given Degree Sequence." Combin. Probab. Comput. 7 (1998), 295-305.

[Newman et al. 00] M. E. J. Newman, S. H. Strogatz, and D. J. Watts. "Random Graphs with Arbitrary Degree Distribution and their Applications." Available from the World Wide Web: (http://xxx.lanl.gov/abs/cond-mat/0007235), 2000.

[Pareto 1897] V. Pareto. Cours d'economie politique. Lausanne et Paris: Rouge, 1897.

[Zipf 49] G. K. Zipf. Human Behaviour and the Principle of Least Effort. New York: Hafner, 1949.

Fan Chung, University of California at San Diego, La Jolla, CA 92093 (fan@euclid.ucsd.edu) Linyuan Lu, University of California at San Diego, La Jolla, CA 92093 (llu@math.ucsd.edu)

Received October 30, 2002; accepted December 11, 2002 
